\title{
Prevalence and predictors of an out-of-range cuff pressure of endotracheal tube in mechanically ventilated patients in a teaching hospital in Iran
}

\author{
Hamidreza Hemmati ${ }^{1,2}$, Shahrzad Izadi ${ }^{1,2}$, Setareh Soltani ${ }^{2,1,3}$, Fatemeh Aghamohammadlou ${ }^{2}$, \\ Majid Mirmohammadkhani ${ }^{4,5^{*}}$
}

1. Department of surgery, Semnan University of Medical Sciences, Semnan, Iran.

2. Kosar Hospital Clinical Research Development Unit, Semnan University of Medical Sciences, Semnan, Iran.

3. Cancer Research Center, Semnan University of Medical Sciences, Semnan, Iran.

4. Social Determinants of Health Research Center, Semnan University of Medical Sciences, Semnan, Iran.

5. Department of Epidemiology and Biostatistics, Semnan University of Medical Sciences, Semnan, Iran.

*Correspondence: Majid Mirmohammadkhani, MD, PhD, E-mail: majidmirmohammadkhani@yahoo.com, ORCID: https://orcid.org/0000-0001-6251-7484

\section{Abstract}

Background: The study aimed to investigate the out-of-range cuff pressure of endotracheal tube in mechanically ventilated patients.

Methodology: This cross-sectional study was performed in Semnan, Iran in 2019. The cuff pressure was measured in patients aged over 18 years who were intubated for at least 24 hours. Out-of-range pressure was defined as a pressure of $<20 \mathrm{cmH}_{2} \mathrm{O}$ or $>30 \mathrm{cmH}_{2} \mathrm{O}$. A multiple logistic regression model was used for analysis.

Results: Of 222 cases, 67 (30.2\%) had cuff pressure in the normal range and 155 (69.8\%) were not in the normal range; $41(18.5 \%)$ and $114(51.4 \%)$ had low and high pressures. The two groups were not significantly different in terms of age and gender. Longer mechanical ventilation $(p=0.004)$ and hospitalization place $(p=0.001)$ were the two factors that had a relationship with the out-of-range pressure. The probability of out-of-range pressure was significantly higher in the cardiac $(p=0.001, O R=6.91)$ and internal medicine $(p=0.008, O R=4.61)$, compared to the emergency intensive care unit.

Conclusion: The authorities should take into consideration some important factors, such as the lack of instructions in hospital units, lack of proficiency for correct measurement, deficient required facilities, and not using the facilities for regular monitoring.

Keywords: Mechanical Ventilation, Endotracheal Intubation, Airway Management, Pressure.

Citation: Hemmati H, Izadi S, Soltani S, Aghamohammadlou F, Mirmohammadkhani M. Prevalence and predictors of an out-of-range cuff pressure of endotracheal tube in mechanically ventilated patients in a teaching hospital in Iran. Anaesth. pain intensive care 2020;24(5):503-508

Received: 4 June 2020, Reviewed: 9 June, 5 July 2020, Accepted: 14 August 2020

\section{Introduction}

Maintaining an airway is the first therapeutic step for all patients in life-threatening conditions, loss of consciousness, and respiratory problems. ${ }^{1}$ Nowadays, considering the improved facilities for taking care of patients, the number of cases requiring endotracheal tube (ETT) has augmented. Exclusively in the United States of America, 13-20 million ETTs are placed yearly. $^{2}$

Endotracheal intubation is performed to maintain an airway and ensure sufficient ventilation. However, 
dangerous and sometimes irreversible complications are similar to other invasive treatment practices. ${ }^{3,4}$ One of the most important side effects is damage to the tracheal mucous due to the excessive dilation of tube cuff leading to erosion, inflammation, the softening of the tracheal rings, tracheal dilation, hemorrhage, infection, fistula, and tracheal stenosis. On the other hand, the insufficient dilation of ETT cuff results in the pulmonary aspiration of upper respiratory airways secretions or air leakage. ${ }^{1,-8}$ To prevent these sequels, the internal pressure of ETT cuff needs to be adjusted so that despite sufficiency, it is not higher than the blood pressure of tracheal wall capillaries. ${ }^{5}$ In standard conditions, this pressure should be $20-30 \mathrm{cmH}_{2} \mathrm{O}{ }^{6}$

Generally, two methods are applied for stabilizing and monitoring ETT cuff pressure in intensive care units. The first technique is completely empirical consisting of touching to control cuff pressure and listening to ensure the lack of air leakage. The second method is measuring by manometer used in more equipped centers. ${ }^{9}$ Besides, the type and quality of the tube are of importance. ${ }^{10}$

To achieve the optimum breathing condition in a patient and prevent the side effects of intubation, the ETT should be regularly controlled and adjusted to standard conditions in patients hospitalized in the special care units. ${ }^{11,12}$ The compilation of guidelines in this regard needs awareness concerning the existing situation in the health centers. Considering the present conditions in the hospitals of Iran and the importance of this subject, the current study aimed to evaluate ETT cuff pressure in mechanically ventilated patients in the special care units of one of the referral teaching hospitals in Iran.

\section{Methodology}

This cross-sectional study was carried out in Kosar referral teaching hospital, Semnan, Iran. Ethical approval was obtained via IR.SEMUMS.REC.1398.19.

Data collection was completed during the first four months of 2019. The study population entailed all the patients aged over 18 years hospitalized in four special care wards, namely emergency, surgery, internal medicine, and cardiac care units during the four-month study course and were under mechanical ventilation for at least $24 \mathrm{~h}$. other inclusion criteria encompassed using ETT with cuff (Size: Fr32, I.D: 8 mm, O.D: 10.7 $\mathrm{mm}$ ) made by HoongAn company and head being at the position of $30-45^{\circ}$. Patients with tracheostomy and cases separate from the ventilation system were not included in the investigation.

The ethical code of IR.SEMUMS.REC.1398.19 and other needed licenses were received. Data were extracted and collected by a thorough review of the history and documents of the patients and interview with the companion if needed. The data included age, gender, ventilation duration (day), body mass index, hospitalization unit, and hospitalization reason (trauma or non-trauma). Afterward, the cuff pressure was measured and recorded utilizing the Mallinckrodt manometer (Covidien, Ireland) in the presence of a unit nurse.

According to the standards of care for mechanically ventilated patients, normal suitable cuff pressure was considered as 20-30 $\mathrm{cmH}_{2} \mathrm{O}$. Cuff pressure in each patient was measured only once randomly in one of the shifts of the morning (8-14), noon (14-20), or night (20-8). Regarding the ethical considerations, in case of cuff pressure was not in the normal range, the needed modification was performed by the unit nurse and was recorded in the documents.

Considering the prevalence of $80 \%-85 \%$ for out-ofrange cuff pressure based on the study conducted by Saleh Moghaddam et al. and the maximum type I error of 0.05 , the sample size was calculated as 220 participants. The normally distributed variables were described by mean and standard deviation and the factors with non-normal distribution were described using the median and interquartile range. Also, the number and percentage were applied for reporting qualitative variables.

The quantitative variables were compared between the two groups of normal and out-of-range pressure using the independent t-test and the Mann-Whitney U test. Moreover, the distribution of qualitative factors between the two groups was compared by the ChiSquare test. The relationship of the variables with cuff pressure was evaluated utilizing the multiple logistic regression and the level of relationship was assessed by the adjusted odds ratio. All the data were analyzed using the Stata software version 11. P-value $<0.05$ was considered significant for all tests. 


\section{Results}

The participants included 120 female and 102 male patients with a mean age of $63.2 \pm 23.8$ years. Most of the cases $(79.7 \%)$ were hospitalized and under mechanical ventilation in one of the special units due to non-traumatic reasons. The mean of cuff pressure in the participants was $15.2 \pm 33.8 \mathrm{cmH}_{2} \mathrm{O}$. According to the findings, the cuff pressure of 67 cases $(30.2 \%)$ was in the normal range and 155 patients $(69.8 \%)$ had outof-range pressure. Among the out-of-range subjects, $41(18.5 \%)$ and $114(51.4 \%)$ of the cases had low and high pressures, respectively (Table 1).

Table 1: Means of cuff pressures and frequency distribution for each range

\begin{tabular}{lcc} 
Range & Mean \pm SD & N (\%) \\
\hline Low pressure & $2.40 \pm 15.4$ & $41(18.5)$ \\
\hline Normal pressure & $3.74 \pm 24.9$ & $67(30.2)$ \\
\hline High pressure & $11.48 \pm 45.7$ & $114(51.4)$ \\
\hline All & $15.23 \pm 33.8$ & $222(100)$ \\
\hline All & $15.23 \pm 33.8$ & $222(100)$ \\
\hline High pressure & $11.48 \pm 45.7$ & $114(51.4)$ \\
\hline All & $15.23 \pm 33.8$ & $222(100)$ \\
\hline
\end{tabular}

The two groups of normal and out-of-range pressure were not significantly different in terms of age $(\mathrm{p}=$ 0.612 ) and gender $(p=0.207)$. The duration of hospitalization with mechanical ventilation had a range of $24 \mathrm{~h}$ to 43 days. The median (and interquartile range) of mechanical ventilation time in patients with out-of-range pressure was 3(5) days and in cases with normal pressure was 1.5(5) days.

The patients with out-of-range pressure had longer mechanical ventilation than the normal pressure $88.4 \%$ group ( $\mathrm{p}<0.001$ ). A significant difference was observed between the units regarding the percentage of out-of-range cuff pressure cases $(\mathrm{p}<0.001)$ with a minimum of $41.7 \%$ and a maximum of $88.4 \%$ in the emergency and cardiac care units, respectively.

Longer mechanical ventilation $(\mathrm{p}=0.004)$ and hospitalization ward $(\mathrm{p}=0.001)$ were the only factors that had a relationship with the augmented incidence of out-of-range pressure (Table 2). The probability of out-of-range cuff pressure was significantly higher in the participants hospitalized in the cardiac care unit $(\mathrm{p}$ $=0.001, \mathrm{OR}=6.91)$ and internal medicine $(\mathrm{p}=0.008$, $\mathrm{OR}=4.61)$, compared to the emergence ward. No

Table 2: The relationship between patients' conditions with an out-of-range cuff pressure of endotracheal tube based on results of multiple logistic regression analysis

\begin{tabular}{|c|c|c|c|c|c|c|c|c|}
\hline \multirow{2}{*}{\multicolumn{2}{|c|}{ Conditions }} & \multicolumn{3}{|c|}{ Count(\%) or Mean \pm SD } & \multirow{2}{*}{ OR } & \multicolumn{2}{|c|}{$95 \% \mathrm{Cl}$} & \multirow{2}{*}{$\mathbf{P}$} \\
\hline & & All & Normal & Out-of-range & & lower & upper & \\
\hline \multicolumn{2}{|l|}{ Age (year) } & $63.2 \pm 23.8$ & $60.2 \pm 24.27$ & $64.4 \pm 23.33$ & 1.01 & 0.98 & 1.02 & 0.573 \\
\hline \multicolumn{2}{|c|}{ BMI $\left(\mathrm{kg} / \mathrm{m}^{2}\right)$} & $25.2 \pm 4.96$ & $24.7 \pm 4.76$ & $25.4 \pm 5.07$ & 1.00 & 0.93 & 1.08 & 0.888 \\
\hline \multicolumn{2}{|c|}{ Vent duration (day) } & $3(5)^{\star}$ & $1.5(2)^{*}$ & $3(5)^{*}$ & 1.24 & 1.07 & 1.44 & 0.004 \\
\hline \multirow{2}{*}{ Gender } & Female & 120 & $39(32.5)$ & $81(67.5)$ & 0.95 & 0.46 & 1.98 & 0.901 \\
\hline & Male & 102 & $28(27.5)$ & $74(72.5)$ & 1 & - & - & - \\
\hline \multirow{3}{*}{ Shift } & Morning & 74 & $21(28.4)$ & $53(71.6)$ & 0.921 & 0.41 & 2.05 & 0.546 \\
\hline & Noon & 74 & $24(32.4)$ & $50(67.6)$ & 0.783 & 0.35 & 1.73 & 0.841 \\
\hline & Night & 74 & $22(29.7)$ & $52(70.3)$ & 1 & - & - & - \\
\hline \multirow{4}{*}{ Care unit } & Cardiac & 69 & $8(11.6)$ & $61(88.4)$ & 6.91 & 2.19 & 21.74 & 0.001 \\
\hline & Internal & 60 & $11(18.3)$ & $49(81.7)$ & 4.61 & 1.47 & 14.37 & 0.008 \\
\hline & Surgical & 69 & $34(49.3)$ & $35(50.7)$ & 1.34 & 0.43 & 4.20 & 0.617 \\
\hline & Emergency & 24 & $14(58.3)$ & $10(41.7)$ & 1 & - & - & - \\
\hline \multirow{2}{*}{ Cause } & trauma & 45 & $17(37.8)$ & $28(62.2)$ & 1.31 & 0.41 & 4.13 & 0.649 \\
\hline & non-trauma & 177 & $50(28.2)$ & $127(71.8)$ & 1 & - & - & - \\
\hline
\end{tabular}

SD: Standard deviation, OR: Adjusted Odds Ratio, Cl: Confidence interval, BMI: Body mass index, Vent: Ventilation, ${ }^{\star} M e d i a n$ (Interquartile range) 
significant difference was reported between the surgery care unit and the emergency ward in terms of normal or out-of-range pressure $(\mathrm{p}=0.617)$.

\section{Discussion}

In the present study, approximately $70 \%$ of the measurements reported out-of-range cuff pressure. Although this rate is similar to other studies in Iran, it is much more prevalent than the international reports. ${ }^{9}$, 13-15 This finding confirms the improper or insufficient monitoring of ETT cuff pressure in the hospitals of Iran.

Further evaluations of the hospitals of Iran demonstrates that there are no serious guidelines concerning the continuous measurement of cuff pressure in patients under mechanical ventilation. On the other hand, measurement is mainly performed empirically through touching and listening. The novel methods, which are not dependent on personal experience are not used much due to the need for facilities (i.e., standard manometers).

In a study conducted on adult patients hospitalized in special care units of hospitals in South Africa, they found that $32 \%$ of nurses checked cuff pressure regularly every $2-4 \mathrm{~h}, 52 \%$ assessed every 6-12 h, and $15 \%$ monitored only when leakage occurred. Moreover, they observed that $1 \%$ of nurses never checked cuff pressure. ${ }^{7}$

Numerous factors have been reported to affect cuff pressure changes, some of which are related to patient conditions and others are related to the monitoring process. $^{9,} 14,16-18$ One of the notable variables in our investigation was the duration of intubation, which was also reported in the previous studies. $4,8,14,19$

The porosity and depreciation generated in ETT cuff during time lead to reduced cuff pressure. Also, other factors such as decreased dose of sedative increased cough, and more consequences due to the discordance of a patient with a ventilator can augment airway pressure resulting in the elevation of cuff pressure during the time.

The increase in the possibility of sequels, such as micro-aspiration and tracheal stenosis during time could be partly attributed to an alteration in cuff pressure during the intubation period. ${ }^{3,9,20}$ Therefore, continuous monitoring of cuff pressure, especially in patients who have ETT for a long time, should be taken into consideration.

Our results indicated that regardless of intubation length, hospitalization units were significantly different in terms of out-of-range pressure prevalence. In this regard, we found that patients hospitalized in internal medicine and cardiac care units had a higher rate of out-of-range pressure, in comparison with surgery and emergency units.

The latter finding might show the difference in the attitude or performance of doctors and nurses of the wards toward monitoring cuff pressure in mechanically ventilated patients. No relationship was observed between the time of measuring (the working shift of nurses) and out-of-range cuff pressure. A review of the literature did not reveal any comparable and similar evidence.

We observed that in more than half of the cases, cuffs had a pressure higher than normal (51.4\%). This result confirms the previous investigations and can highly be related to the methods and guidelines of pressure monitoring. A review study concluded that the common empirical techniques for monitoring ETT cuff pressure cause the pressure to be higher than the normal range. ${ }^{8,9}$ Methods of measuring a manometer should replace empirical approaches by providing the required facilities.

In the present study, no relationship was revealed between age, gender, hospitalization reason, and outof-range cuff pressure, which is in line with the results of previous studies. ${ }^{12,13,18,20}$ Furthermore, we did not observe any relationship between body mass index and out-of-range pressure, which is consistent with some other investigations. ${ }^{12,} 18$ However, Taslimi et al. reported a correlation between the two mentioned variables. $^{20}$ This controversy might be due to the elimination of the probable impact of some confounding factors, including hospitalization duration in the present study and similar investigations.

Saleh Moghaddam et al. indicated a significant relationship between body temperature and cuff pressure, while did not find any relationship between tube type and cuff pressure. ${ }^{13}$ In the present study, body temperature was not controlled and this should be taken into consideration for interpreting the results. Moreover, it should be noted that a similar tube type 
was applied to all patients during the study period. Another limitation of the current investigation was that we evaluated neither pregnant patients nor cases aged under 18 years.

Recent studies recommended modifying interventions in the process of monitoring ETT cuff pressure. ${ }^{21,22}$ According to the findings of the present study, modifications in cuff pressure monitoring are highly needed and recommended. To prevent the complications of out-of-range cuff pressure (e.g., aspiration, stenosis, and fistula) training and eliminating the background problems, such as the lack of experience and skill for correct measuring among personnel, insufficient facilities, and not regularly using the facilities for monitoring are recommended. Furthermore, an accurate guideline for regular pressure monitoring along with pressure measurement and modification at least once at each working shift seems to be necessary.

\section{Conclusion}

According to the results of this study, the prevalence of out-of-range cuff pressure was very high. The present study was completed in a teaching hospital and more problems are expected in non-teaching hospitals. Our findings revealed that effective intervention for modifying the procedure of taking care of patients with ETT in terms of monitoring ETT cuff pressure seems necessary in hospitals of Iran.

\section{Funding}

The present research was supported by Semnan University of Medical Sciences.

\section{Conflict of interests}

No Conflicts of interest/Competing interest was declared by the authors.

\section{Authors' contribution}

HH, SI, SS: Concept, conduct of the study work and manuscript editing

FA: Concept, literature search, conduct of the study work and manuscript editing

MM: Concept, literature search, statistical analysis and manuscript editing

\section{References}

1. Morris LL, Whitmer A, Mclntosh E. Tracheostomy care and complications in the intensive care unit. Crit Care Nurse. 2013 Oct;33(5):18-30. [PubMed] DOI: $10.4037 / \operatorname{ccn} 2013518$

2. Sultan P, Carvalho B, Rose BO, Cregg R. Endotracheal tube cuff pressure monitoring: a review of the evidence. J Perioper Pract. 2011 Nov;21(11):379-86. [PubMed] DOI: $10.1177 / 175045891102101103$

3. Efrati S, Deutsch I, Gurman GM. Endotracheal tube cuff--small important part of a big issue. J Clin Monit Comput. 2012 Feb;26(1):53-60. [PubMed] DOI: $10.1007 / \mathrm{s} 10877-011-9333-\mathrm{x}$

4. Hemmati $H$, Forozeshfard $M$, Hosseinzadeh $B$, Hemmati S, Mirmohammadkhani M, Bandari R. Tracheostomy in Patients Who Need Mechanical Ventilation: Early or Late? Surgical or Percutaneous? A Prospective Study in Iran. Indian J Surg. 2017 Oct;79(5):406-411. [PubMed] [Free Full Text] DOI: 10.1007/s12262-016-1497-7

5. Sole ML, Poalillo FE, Byers JF, Ludy JE. Bacterial growth in secretions and on suctioning equipment of orally intubated patients: a pilot study. Am J Crit Care. 2002 Mar;11(2):141-9. [PubMed]

6. El-Orbany M, Salem MR. Endotracheal tube cuff leaks: causes, consequences, and management. Anesth Analg. 2013 Aug;117(2):428-34. [PubMed] DOI: 10.1213/ANE.0b013e318292ee21

7. Jordan $P$, Van Rooyen D, Venter D. Endotracheal tube cuff pressure management in adult critical care units. Southern African J Crit Care. 28 (1):13-16

8. Nikbakhsh N, Alijanpour E, Mortazavi $\mathrm{Y}$, Organji N (2010) Evaluation of Tracheal Tube Cuff Pressure Complications in ICU Patients of Shahid Beheshti hospital. JBUMS 2010, 12(2): 30-34. [Free Full Text]

9. Grant T. Do current methods for endotracheal tube cuff inflation create pressures above the recommended range? A review of the evidence. J Perioper Pract. 2013 Dec;23(12):292-5. DOI: $\underline{10.1177 / 175045891302301205}$

10. Young PJ, Pakeerathan S, Blunt MC, Subramanya S. A low-volume, low-pressure tracheal tube cuff reduces pulmonary aspiration. Crit Care Med. 2006 Mar;34(3):632-9. [PubMed] DOI: 10.1097/01.CCM.0000201406.57821.5B

11. Nseir S, Duguet A, Copin MC, De Jonckheere J, Zhang $\mathrm{M}$, Similowski T, Marquette $\mathrm{CH}$. Continuous control of endotracheal cuff pressure and tracheal wall damage: a randomized controlled animal study. Crit Care. 
2007;11(5):R109. [PubMed] [Free Full Text] DOI: $10.1186 / c c 6142$

12. Liu J, Zhang X, Gong W, Li S, Wang F, Fu S, Zhang M, Hang Y. Correlations between controlled endotracheal tube cuff pressure and postprocedural complications: a multicenter study. Anesth Analg. 2010 Nov;111(5):1133-7.

[PubMed] DOI: 10.1213/ANE.0b013e3181f2ecc7

13. Saleh Moghaddam A, Malekzade J, Mesbahi Z, Esmaeli $H$ (2013) Relationship between temperature and cuff pressure in mechanically ventilated patients with endotracheal tube. The Horizon of Medical Sciences 19 (2):105-109 [Free Full Text]

14. Nasiri E, Mohamadpoor R, Mortazavi Y, Khorrami M. A comparison change in endotracheal tube cuff pressure between air and Lidocaine and N2O with $\mathrm{O} 2$ cuff inflation during general anesthesia. J Gorgan Univ Med Sci 2004, 6(2): 32-39 (2):32-39 [Free Full Text]

15. Nseir $\mathrm{S}$, Brisson $\mathrm{H}$, Marquette $\mathrm{CH}$, Chaud P, Di Pompeo C, Diarra M, Durocher A. Variations in endotracheal cuff pressure in intubated critically ill patients: prevalence and risk factors. Eur J Anaesthesiol. 2009 Mar;26(3):229-34. [PubMed] DOI: 10.1097/eja.0b013e3283222b6e

16. Valencia M, Ferrer M, Farre R, Navajas D, Badia JR, Nicolas JM, Torres A. Automatic control of tracheal tube cuff pressure in ventilated patients in semirecumbent position: a randomized trial. Crit Care Med. 2007 Jun;35(6):1543-9.

[PubMed]
17. Sanaie S, Rahmani F, Chokhachian S, Mahmoodpoor A, Rahimi Panahi J, Mehdizadeh Esfanjani R, Mirzaei $\mathrm{M}$, Soleimanpour H. Comparison of tracheal tube cuff pressure with two technique: fixed volume and minimal leak test techniques. J Cardiovasc Thorac Res. 2019;11(1):48-52. [PubMed] [Free Full Text] DOI: $10.15171 /$ jcvtr.2019.08

18. Hoffman R, Parwani V, Kaban J, Dueffer H, Howell A, Sturmann K. Comparison of Two Common Techniques for Inflating Endotracheal Tube Cuffs: Set Volume of Air Vs. Palpation of the Pilot Balloon. Ann Emerg Med 48 (4):27 DOI: 10.1016/j.annemergmed.2006.07.534

19. Alzahrani AR, Al Abbasi S, Abahoussin OK, Al Shehri TO, Al-Dorzi HM, Tamim HM, Sadat M, Arabi YM. Prevalence and predictors of out-of-range cuff pressure of endotracheal and tracheostomy tubes: a prospective cohort study in mechanically ventilated patients. BMC Anesthesiol. 2015 Oct 15;15:147. [PubMed] [Free Full Text] DOI: 10.1186/s12871-015-0132-7

20. Taslimi L, Ghanbari A, Kazemnezhad Leili E . Study of endotracheal tube cuff pressure and time of measurement among intensive care units patients. J Holist Nurs Midwifery 26 (2):29-37

21. Sole ML, Su X, Talbert S, Penoyer DA, Kalita S, Jimenez E, Ludy JE, Bennett M. Evaluation of an intervention to maintain endotracheal tube cuff pressure within therapeutic range. Am J Crit Care. 2011 Mar;20(2):109-17; quiz 118. [PubMed] [Free Full Text] DOI: 10.4037/ajcc2011661 